\section{UJMM

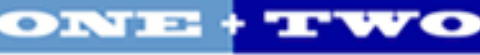

\section{Undergraduate Journal of Mathematical}

Modeling: One + Two

2020

\title{
Prosthetic Leg Model
}

Dang Nguyen

University of South Florida

Advisors:

Arcadii Grinshpan, Mathematics and Statistics

Dmitri Voronine, Physics

Problem Suggested By: Dmitri Voronine

Field of Study for Problem Suggester: Physics

Follow this and additional works at: https://digitalcommons.usf.edu/ujmm

Part of the Mathematics Commons

UJMM is an open access journal, free to authors and readers, and relies on your support:

Donate Now

\section{Recommended Citation}

Nguyen, Dang (2020) "Prosthetic Leg Model," Undergraduate Journal of Mathematical Modeling: One +

Two: Vol. 10: Iss. 2, Article 2.

DOI: https://doi.org/10.5038/2326-3652.10.2.4915

Available at: https://digitalcommons.usf.edu/ujmm/vol10/iss2/2 


\title{
Prosthetic Leg Model
}

\begin{abstract}
The main goal of this paper is to introduce an imitated prosthetic leg model by analyzing the applied forces. Even though the model is based on the idea of a prosthetic leg, it is also applicable to people without disabilities. The same concept of the model can be seen in the circus, where a person maintains a balanced state while on an incredible height without falling. When all the applying forces in the system are calculated, the design achieves the ideal state which allows it to function most effectively. One of the essential factors applied to the imitated prosthetic leg model, that causes a great impact on the human body's movement, is torque. Torque is proportional to the spinning force that tends to cause rotation about a certain axis. Therefore, the paper also focuses on the idea of moment of inertia and center of mass to estimate torque. The density of different materials is also considered and compared in order to provide insights as to which would be the most effective.
\end{abstract}

Keywords

prosthetic leg, torque, moment of inertia, center of mass

Creative Commons License

(c) $(i) \Theta(9$

This work is licensed under a Creative Commons Attribution-Noncommercial-Share Alike 4.0 License. 


\section{PROBLEM STATEMENT}

Based on the idea of how a prosthetic leg operates, we analyze and calculate forces that apply to the new model in movement and, then, make the adjustment to its length and material for it to be applied in real life. We also consider an environmental condition when the model travels through a liquid.

\section{MOTIVATION}

Newtonian forces play a crucial role not only in science and engineering but also provide us with countless applications on a daily basis. According to classical physics, suitable to describe the macroscopic world, the motion of any object in the universe is not random but rather quantifiable, calculable, and therefore predictable. As a result, the movement of objects and humans can also be analyzed and calculated by considering the effects of the applied forces. Therefore, it is essential to be able to understand the nature of the forces and what factors influence the movement of the object. This paper examines one example which includes a variety of forces: normal, gravitational, buoyancy, drag, and static friction, all of which affect a certain rotating object. It also applies the idea of moment of inertia and center of mass in order to illustrate precisely the rotational effect resulting from the aforementioned forces. All of them are interpreted based on the classical mechanics and Newton's second law of motion.

\section{MATHEMATICAL DESCRIPTION AND SOLUTION APPROACH}

\section{A) ANALYZING FORCES}

First of all, we need to analyze all the equations that are important in explaining how the model functions. The main theme of this paper utilizes Newton's second law of motion: 


$$
\sum F=M a
$$

This equation predicts the movement of the model under the condition when the forces applied on it are unbalanced. We will take normal, gravitational, buoyancy, drag and static frictional forces into consideration when calculating the applied forces, namely:

$$
\begin{aligned}
& N=M g \cos (x) \\
& F_{g}=M g \\
& F_{B}=M_{\text {fluid }} * g \\
& F_{D}=\frac{1}{2} \rho v^{2} C_{D} A \\
& f_{S}=\mu_{s} * N
\end{aligned}
$$

Equations (1) and (2) represent the forces that we and most of the objects experience on a daily basis, i.e. normal and gravitational forces. The variable " $x$ " is the angle of incline since the normal force only exerts an action perpendicular to the surface, " $g$ " is the gravitational acceleration which approximately is given by $9.81 \mathrm{~m} / \mathrm{s}^{2}$ and $M$ is the mass of the object itself. The equation illustrates how we are constantly affected by the earth and the surface that we are standing on. Equation (3) depicts the buoyancy force that is created by a fluid (such as an object crosses a river), where $M_{\text {fluid }}$ is the mass of fluid that the object displaces and it can be presented by:

$$
\rho=\frac{M_{\text {fluid }}}{V},
$$

where $\rho$ is the density of freshwater, $V$ is the volume of water that the object displaces.

We consider freshwater for river, with density approximately equal to $1000 \mathrm{~kg} / \mathrm{m}^{3}$ and without any viscosity. To calculate the drag force by the river, we apply equation (4) that still uses " $\rho$ " for freshwater and where $C_{D}$ is the drag coefficient that depends on the material. Lastly, we have the static friction. Equation (5) is included in order to prevent a user from slipping, and the static coefficient depends on two factors: the surface that our model travels on and the material of the model itself. 

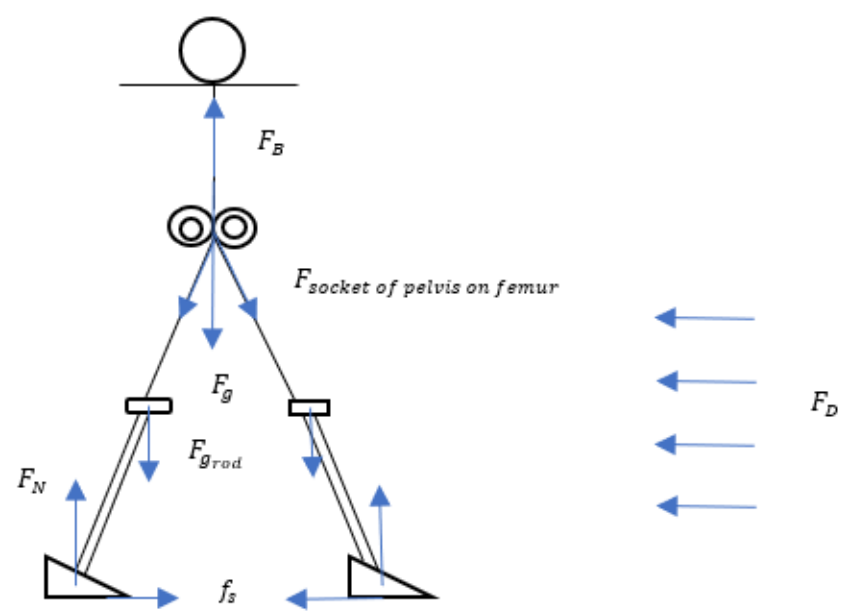

Figure 1: Different forces apply on human body when using the model

Before we can analyze the forces affecting a moving object, which in this case is the human leg combined with the designed model when a person is walking, it is important to understand the mechanism of the human leg's movement which is called the gait cycle. We will only consider two main phases and analyze the applied forces: "Mid stance" for both legs in phase 1 and “Terminal swing” for one leg in phase 2. Chakravarthy, Sultana, Srinath, and Kumar conducted a research in 2017 that culminated in the paper "A mathematical model to determine the torque for a prosthetic leg - Lagrangian equation", in which this cycle is illustrated:

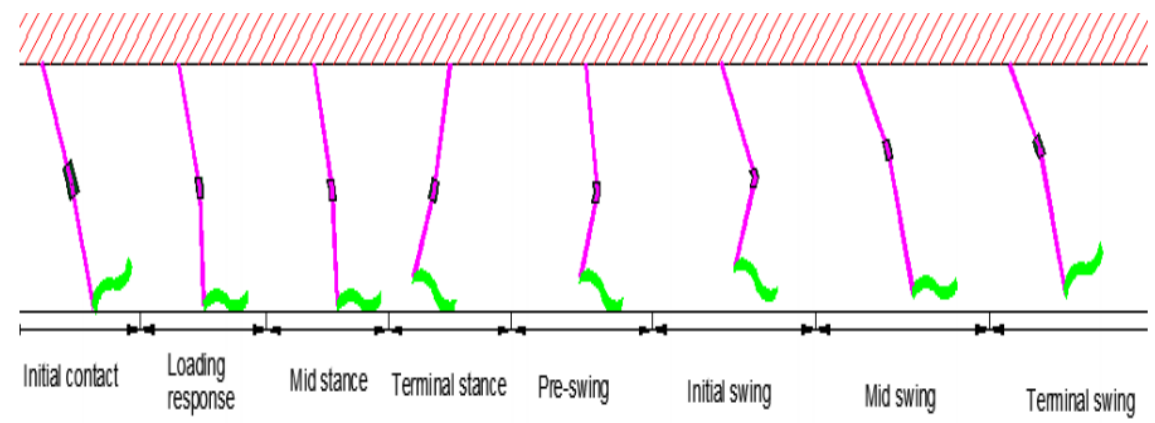

Figure 2: Gait cycle (Chakravarthy, Sultana, Srinath and Kumar, 2017, p.52) 
$1^{\text {st }}$ Phase of the movement:

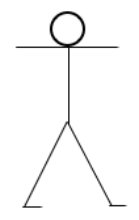

Figure 3: both legs in "Mid stance" position

$2^{\text {nd }}$ Phase of the movement:

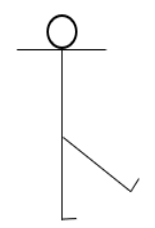

Figure 4: One leg in "Mid stance" and one leg in "Terminal swing" position

These two phases represent the main movement when using the design. Note that we already combine the human leg and the model as a "straight rod" having neglected the rotational motion at the knee. Moreover, there are forces on the vertical direction such as normal, gravitational and buoyancy forces which can be balanced out (acceleration in $\hat{\imath}=0$ ). Because of that, we will shift our attention to the forces in the horizontal direction such as drag and frictional forces (Numerical value will be used in part C -Application- to make actual calculations for the functional model).

Based on this idea, we can estimate the magnitude of the outcome forces on the model if there is enough data. However, since the human leg is rather rotational motion than translational motion, i.e. it rotates around the hip joint; we also need to take torque into account. The main objective is to calculate all the forces and estimate the range for ideal torque so that a human can use the model effectively, since the human hip joint can only exert in practice a certain amount of 
torque. For the sake of simplicity, we only consider torque at the hip joint and neglect the rotational motion in the knee by considering the prosthetic leg and real human leg combined as one straight, circular "rod":

$$
\begin{gathered}
\tau=F * r, \\
\tau=\mathrm{I} * \alpha,
\end{gathered}
$$

where "I" stands for the moment of inertia, which can be calculated by the following equation, in which $m$ is mass of the object and $r$ is the distance from the mass to its axis of rotation:

$$
\mathrm{I}=\sum_{i=1}^{N} m_{i} r_{i}^{2}
$$

This equation is mainly used for calculating the moment of inertia of the system where there are more than one mass interconnected with each other. To be able to estimate the moment of inertia for this model, we need to reform integration. This procedure consists of two parts: the "uniform rod"-combination of the real human leg and the model, and the "base" which serves as a bridge to connect the model with the surface. Rotational axis will be located at the hip joint and the length of "uniform rod" is $L$ :

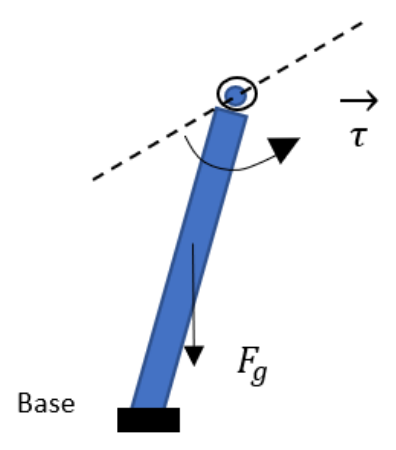

Figure 5: Model illustrates the gravitational force and torque applied on human leg Note: The model's size and weight will be modified so the combination between human leg and the model will get as close to the uniform rod as possible 
We know that the density of the object, based on its dimensions, can be represented as:

$$
\begin{aligned}
& \Rightarrow 1 D \lambda=\frac{m}{\text { length }} \\
& \Rightarrow 2 D \sigma=\frac{m}{\text { Area }} \\
& \Rightarrow 3 D \rho=\frac{m}{\text { Volume }}
\end{aligned}
$$

\section{B) CALCULATIONS}

Part 1: Calculating the moment of inertia for the combination of the model and human leg around the axis of rotation

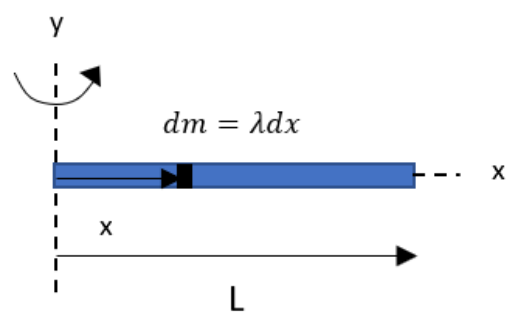

Figure 6: Calculating the moment of inertia for the rod with an axis of rotation at one end

Note: The image is shown in 2 dimensions and the axis of rotation lines along $y$-axis for the observation purpose but the result will be assumed to be the same.

We have: $1 D \lambda=\frac{m}{\text { length }}$

$\mathrm{I}=\int r^{2} d m$, where $x$ can substitute for $r, d m$ can be substituted by $\lambda d x$

$\Leftrightarrow \mathrm{I}=\int_{0}^{L} x^{2} \lambda d x=\lambda \int_{0}^{L} x^{2} d x$

$\Leftrightarrow \mathrm{I}=\frac{\lambda x^{3}}{3}$ from 0 to $L$

$\Leftrightarrow \mathrm{I}_{\text {combination of human leg and the model with the rotational axis at hip joint }}=\frac{m}{L} * \frac{L^{3}}{3}=\frac{m L^{2}}{3}$ 
Part 2: Calculating the moment of inertia for the base

First case: The rectangular prism. When calculating the moment of inertia for the base, we notice that the axis of rotation is in the different location, therefore we need to calculate the moment of inertia that passes through the center of mass of the rectangular prism, and then we apply the parallel axis theorem to shift the axis of rotation to the hip joint. In this specific case, the moment of inertia is not affected whether the base is in $2 \mathrm{D}$ or $3 \mathrm{D}$, therefore, we will integrate the rectangle with the axis passing through its center (the center of mass of a symmetrical shape is always at its center, including rectangle).

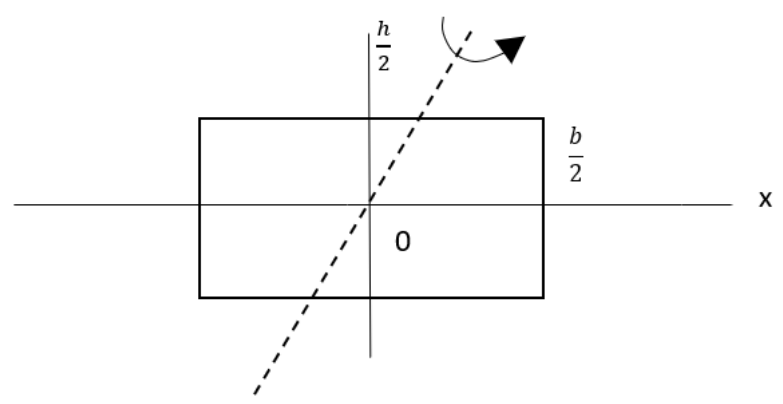

Figure 7: Calculating the moment of inertia for the base with rectangle prism shape

We have: $h$ for height, $b$ for base

$$
\begin{aligned}
& \Rightarrow 2 D \sigma=\frac{m}{\text { Area }} \Leftrightarrow m_{i}=\frac{m}{h b} \\
& \Rightarrow d A=d x^{*} d y \\
& \mathrm{I}= \iint^{2} m r^{2} d A=\int_{-h / 2}^{h / 2} d y \int_{-\frac{b}{2}}^{\frac{b}{2}} d x \frac{m}{h b}\left(x^{2}+y^{2}\right) \\
& \Leftrightarrow \mathrm{I}=\frac{m}{h b}\left(\left.\int_{-h / 2}^{h / 2} \frac{x^{3}}{3} d y\right|_{-b / 2} ^{b / 2}+\left.\int_{-h / 2}^{h / 2} y^{2} x\right|_{-b / 2} ^{b / 2} d y\right) \\
& \Leftrightarrow \mathrm{I}=\frac{m}{h b}\left(\frac{b^{3} y}{3 * 4}\left|\begin{array}{c}
h / 2 \\
-h / 2
\end{array}+\frac{y^{3}}{3} b\right|_{-h / 2}^{h / 2}\right) \\
& \Leftrightarrow \mathrm{I}=\frac{m}{h b}\left(\frac{b^{3} h}{12}+\frac{b h^{3}}{12}\right)
\end{aligned}
$$


$\Leftrightarrow \mathrm{I}=\frac{m}{12}\left(b^{2}+h^{2}\right)$

This is the moment of inertia for the rectangular prism whose rotational axis is right at its centroid, therefore its $\mathrm{I}_{C o M}$ is also equal to $\frac{m}{12}\left(b^{2}+h^{2}\right)$. Now we apply the parallel axis theorem to shift the axis:

$$
\mathrm{I}_{\mathrm{II}}=\mathrm{I}_{\mathrm{CoM}}+m d^{2}
$$

( $\mathrm{I}_{\|}$is the new moment of inertia that has the rotational axis parallel to the moment of inertia at the center of mass, and $\mathrm{d}$ is the distance between the two axes)

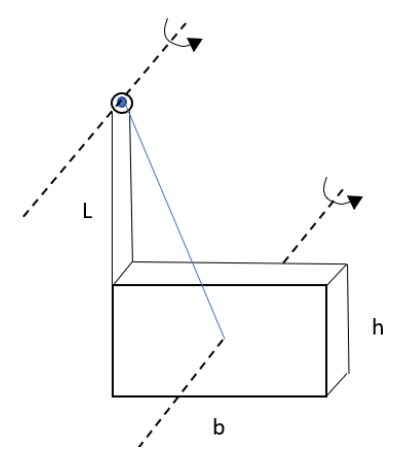

Figure 8: Calculating the moment of inertia for the rectangle prism with axis of rotation shifted

We will use $L$ as the length of the combination of the human leg and the model:

$$
\begin{aligned}
\Rightarrow \mathrm{I}_{\|} & =\frac{m}{12}\left(b^{2}+h^{2}\right)+m\left(\left(\frac{b}{2}\right)^{2}+\left(\frac{h^{2}}{2}+L\right)^{2}\right) \\
& =\frac{m b^{2}}{12}+\frac{m h^{2}}{12}+\frac{m b^{2}}{4}+m\left(\frac{h^{4}}{4}+\frac{2 h^{2}}{2} L+L^{2}\right) \\
& =\frac{m b^{2}}{12}+\frac{m h^{2}}{12}+\frac{m b^{2}}{4}+\frac{m h^{4}}{4}+m h^{2} L+m L^{2} \\
& =\frac{m b^{2}}{3}+\frac{m h^{2}}{12}+\frac{m h^{4}}{4}+m h^{2} L+m L^{2}
\end{aligned}
$$

This is the moment of inertia for the base that has a rectangular prism shape whose axis of rotation passes though the human's hip joint. As a result, the moment of inertia for the whole human leg, which includes the leg, the model and the base, will be: 


$$
\begin{aligned}
\mathrm{I} & =\frac{m b^{2}}{3}+\frac{m h^{2}}{12}+\frac{m h^{4}}{4}+m h^{2} L+m L^{2}+\frac{m L^{2}}{3} \\
& =\frac{m b^{2}}{3}+\frac{m h^{2}}{12}+\frac{m h^{4}}{4}+m h^{2} L+\frac{4 m L^{2}}{3}
\end{aligned}
$$

Second case: The triangular prism. This case is more complicated, but its flexibility is higher than the rectangular prism and therefore requires less material. We will find its moment of inertia by applying the same steps as for the rectangular prism. Since for the triangle the center of mass is unknown, we first apply the following equations to obtain it:

$$
\begin{aligned}
& X_{c m}=\frac{\sum_{i}^{n} m_{i} x_{i}}{\sum_{i}^{n} m_{i}}=\frac{m_{1} x_{1}+m_{2} x_{2}+\cdots+m_{n} x_{n}}{m_{1}+m_{2}+\cdots+m_{n}} \\
& Y_{c m}=\frac{\sum_{i}^{n} m_{i} y_{i}}{\sum_{i}^{n} m_{i}}=\frac{m_{1} y_{1}+m_{2} y_{2}+\cdots+m_{n} y_{n}}{m_{1}+m_{2}+\cdots+m_{n}} \\
& Z_{c m}=\frac{\sum_{i}^{n} m_{i} z_{i}}{\sum_{i}^{n} m_{i}}=\frac{m_{1} z_{1}+m_{2} z_{2}+\cdots+m_{n} z_{n}}{m_{1}+m_{2}+\cdots+m_{n}}
\end{aligned}
$$

Instead of finding $\mathrm{CoM}$ for a 3D shape, the triangular prism, we can find $\mathrm{CoM}$ for the triangle which is two-dimensional, since by reasons of symmetry we know, that the 3D CoM would lie along a line that is perpendicular to the plane we find in two dimensions.

We know that:

$$
X_{c m}=\frac{\int x d m}{\int d m}, Y_{c m}=\frac{\int y d m}{d m}
$$

where $d m$ is the small part of the total mass.

$\Rightarrow$ Calculating $X_{c m}$

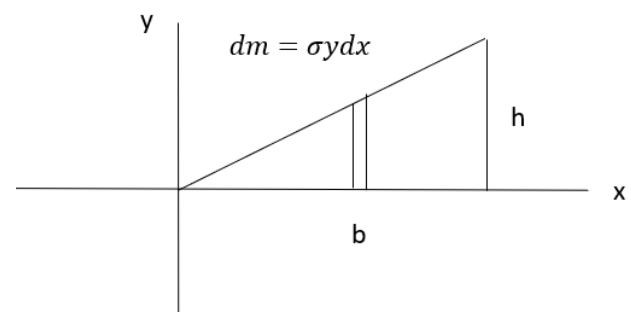

Figure 9: Calculating the center of mass of the triangle in $x$-axis 
$X_{c m}=\frac{\int x d m}{\int d m}=\frac{1}{m} \int_{0}^{b} x d m=\frac{1}{m} \int_{0}^{b} x \sigma y d x$

Since $y=\frac{h x}{b}$

$\Leftrightarrow X_{c m}=\frac{1}{m}\left(\frac{\sigma h}{b}\right) \int_{0}^{b} x^{2} d x=\frac{\sigma h b^{3}}{3 m b}=\frac{\sigma h b^{2}}{3 m}$

$\Leftrightarrow X_{c m}=\frac{m}{\frac{1}{2} b h}\left(\frac{h b^{2}}{3 m}\right)=\frac{2}{3} b$

$\Rightarrow$ Calculating $Y_{c m}$

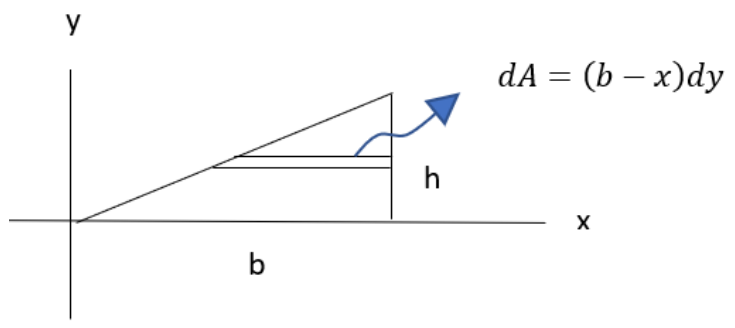

Figure 10: Calculating the center of mass of the triangle in y-axis

$Y_{c m}=\frac{\int y d m}{d m}=\frac{1}{m} \int y d m$

Substitute $d m$ for $\sigma d A$, where $\mathrm{dA}=(\mathrm{b}-\mathrm{x}) \mathrm{dy}$. We also know that $x=\frac{b y}{h}$, therefore

$\Leftrightarrow Y_{c m}=\frac{1}{m} \int y \sigma d A=\frac{1}{m} \int_{0}^{h} y \sigma(b-x) d y$

$\Leftrightarrow Y_{c m}=\frac{1}{m} \sigma \int_{0}^{h} y\left(b-\frac{b y}{h}\right) d y=\frac{1}{m} \sigma\left(\frac{b h^{2}}{2}-\frac{b h^{3}}{3 h}\right)=\frac{1}{m} \sigma \frac{b h^{2}}{6}$

And since $\sigma=\frac{m}{\text { Area }}=\frac{m}{\frac{1}{2} b h}$

$\Leftrightarrow Y_{c m}=\frac{1}{m}\left(\frac{m}{\frac{1}{2} b h}\right) \frac{b h^{2}}{6}=\frac{h}{3}$

We can now obtain the coordinate of the CoM of the triangle based on its base and height:

$\left(\frac{2 b}{3}, \frac{h}{3}\right)$. The axis of rotation passes through this point and also passes through the CoM of the 
triangular prism and it is perpendicular to the object's surface (which means it is in the direction of z-plane).

The second problem of the triangular prism is its axis of rotation. To calculate the moment of inertia in which the axis of rotation is right at the center of mass is complicated. Therefore, we find moment of inertia about the line of rotation in figure 11 , located at $\left(\frac{b}{2}, \frac{h}{2}\right)$, and then apply the parallel axis theorem to find the moment of inertia about the center of mass, and apply the parallel axis theorem for the second time to find the moment of inertia about the axis of rotation located at the human's hip joint.

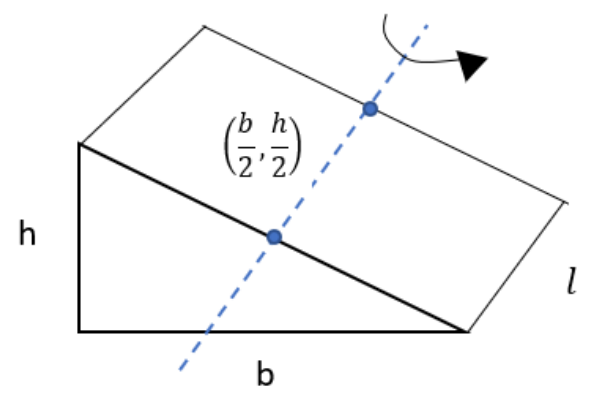

Figure 11: Calculating the moment of inertia for the triangle prism with shifted axis of rotation

Now we integrate the triangular prism with the axis of rotation at $\left(\frac{b}{2}, \frac{h}{2}\right)$

We have $\mathrm{b}$ for base, $\mathrm{h}$ for height and $l$ for length of the triangular prism.

$$
\begin{aligned}
\mathrm{I}_{z} & =\int\left(x^{2}+y^{2}\right) \rho d v \\
& =\rho l \int_{0}^{b} \int_{0}^{h-\frac{h x}{b}}\left(x^{2}+y^{2}\right) d x d y \\
\mathrm{I}_{z} & =\iiint\left(x^{2}+y^{2}\right) \rho d v \\
& =\rho l \int_{0}^{b} \int_{0}^{h-\frac{h x}{b}}\left(x^{2}+y^{2}\right) d x d y \\
\mathrm{I}_{y} & =\left(x^{2} y+\frac{y^{3}}{3}\right) \text { from } 0 \text { to } h-\frac{h}{b} x
\end{aligned}
$$




$$
\begin{aligned}
& =\frac{\left(b-\frac{h x}{b}\right)^{3}}{3}+x^{2}\left(b-\frac{h x}{b}\right) \\
& \Rightarrow \mathrm{I}=\rho l \int_{0}^{b} \mathrm{I}_{y} d x=\frac{b l \rho\left(8 b^{3}-9 b^{2} h+4 b h^{2}-h^{3}\right)}{12}, \text { with } \rho=\frac{m}{\text { Volumn }}=\frac{m}{\frac{1}{2} b h l} \\
& \Rightarrow \mathrm{I}=\frac{m\left(8 b^{3}-9 b^{2} h+4 b h^{2}-h^{3}\right)}{6 h}=\frac{8 m b^{3}}{6 h}-\frac{9 b^{2} h m}{6 h}+\frac{4 b h^{2} m}{6 h}-\frac{h^{3} m}{6 h} \\
& \Rightarrow \mathrm{I}=\frac{4 b^{3} m}{3 h}-\frac{3 b^{2} m}{2}+\frac{2 b h m}{3}-\frac{h^{2} m}{6}
\end{aligned}
$$

To find the moment of inertia with the axis of rotation at CoM, we have to flip the triangle to sync the two triangles together. Therefore the CoM that we will use is at $\left(\frac{b}{3}, \frac{h}{3}\right)$, and now we will find the distance from it to the point $\left(\frac{b}{2}, \frac{h}{2}\right)$ to apply parallel axis theorem for the triangular prism.

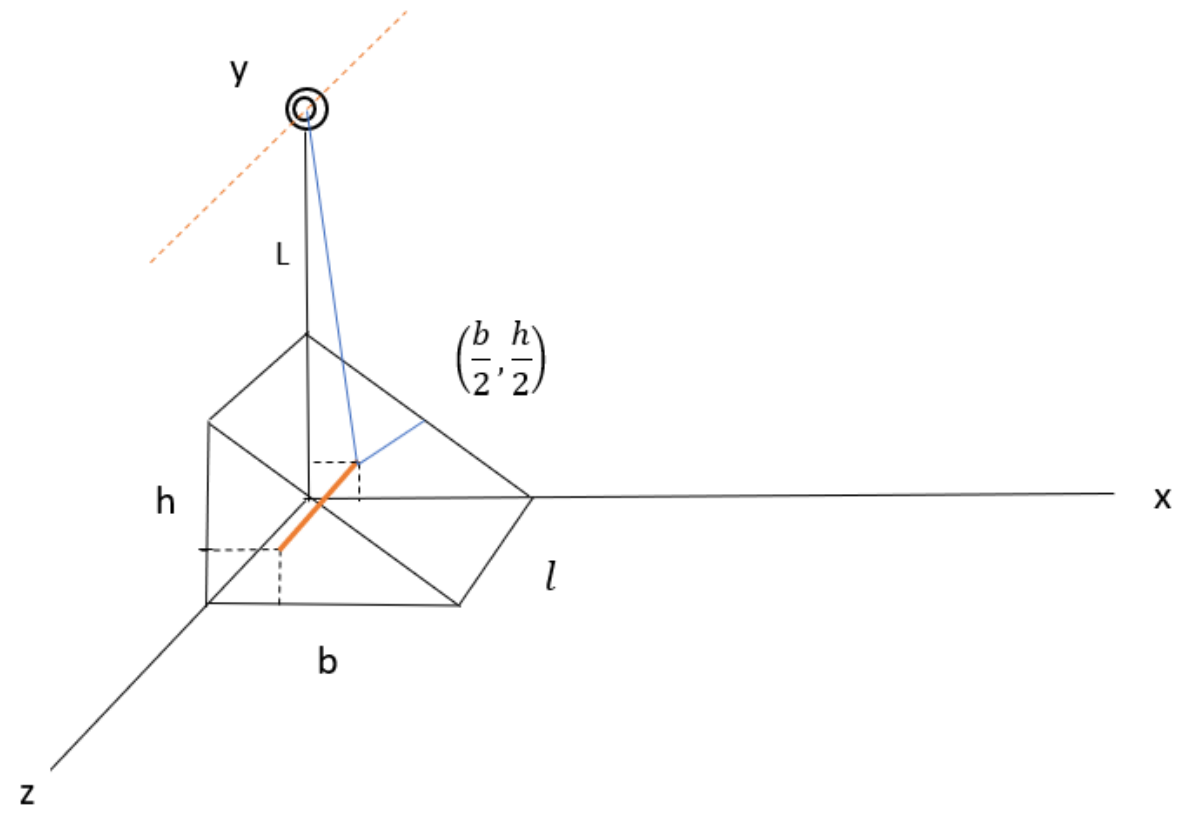

Figure 12: Calculating the moment of inertia for the triangle prism with axis of rotation located at human's hip joint.

Distance from $\left(\frac{b}{3}, \frac{h}{3}\right)$ to $\left(\frac{b}{2}, \frac{h}{2}\right)=\sqrt{\left(\left(\frac{b}{2}-\frac{b}{3}\right)^{2}+\left(\frac{h}{2}-\frac{h}{3}\right)^{2}\right)}$

$=\sqrt{\left(\frac{b}{6}\right)^{2}+\left(\frac{h}{6}\right)^{2}}=\sqrt{\frac{b^{2}+h^{2}}{36}}$ 
$=\frac{\sqrt{b^{2}+h^{2}}}{6}$

Then we apply the parallel axis theorem: $\mathrm{I}_{\|}=\mathrm{I}_{\text {CoM }}+m d^{2}$

$$
\Rightarrow \mathrm{I}_{C o M}=\frac{4 b^{3} m}{3 h}-\frac{3 b^{2} m}{2}+\frac{2 b h m}{3}-\frac{h^{2} m}{6}-\frac{b^{2}+h^{2}}{36}
$$

Distance from CoM of the triangular prism to the human's hip joint can be calculated by:

$\sqrt{\left(L+\frac{2 h}{3}\right)^{2}+\left(\frac{b}{3}\right)^{2}}$

We use $\mathrm{L}$ as the length of the combination of the human leg and the model. Now we apply the parallel axis theorem again to obtain:

$$
\begin{aligned}
\mathrm{I}_{\|} & =\left(\frac{4 b^{3} m}{3 h}-\frac{3 b^{2} m}{2}+\frac{2 b h m}{3}-\frac{h^{2} m}{6}-\frac{b^{2}+h^{2}}{36}\right)+m *\left[\left(L+\frac{2 h}{3}\right)^{2}+\left(\frac{b}{3}\right)^{2}\right] \\
& =\frac{4 b^{3} m}{3 h}-\frac{3 b^{2} m}{2}+\frac{2 b h m}{3}-\frac{h^{2} m}{6}-\frac{b^{2}+h^{2}}{36}+m L^{2}+\frac{4 m L h}{3}+\frac{4 m h^{2}}{9}+\frac{m b^{2}}{9}
\end{aligned}
$$

For the triangular prism we calculate the moment of inertia for the combination of the model, human leg and the base that has axis of rotation at human's hip joint to be equal to:

$$
\begin{aligned}
& \Rightarrow \frac{4 b^{3} m}{3 h}-\frac{3 b^{2} m}{2}+\frac{2 b h m}{3}-\frac{h^{2} m}{6}-\frac{b^{2}+h^{2}}{36}+m L^{2}+\frac{4 m L h}{3}+\frac{4 m h^{2}}{9}+\frac{m b^{2}}{9}+\frac{m L^{2}}{3}= \\
& \frac{4 b^{3} m}{3 h}+\frac{2 b h m}{3}-\frac{b^{2}+h^{2}}{36}+\frac{4 m L h}{3}+\frac{4 m L^{2}}{3}-\frac{25 b^{2} m}{18}+\frac{5 m h^{2}}{18}
\end{aligned}
$$

\section{C) APPLICATION}

In this part, we will take a closer look into the numerical data and calculate the actual range for different values in order to create a functional imitated prosthetic leg model.

Part 1: Estimating the length of the combination between the human leg and the model, which lead to measure its total weight 
According to the previous research (see appendix), we use the following data:

- Average human weight: $75 \mathrm{~kg}$

- Average mass for 1 human leg: 17-19\% of total mass $\rightarrow \sim 13.5 \mathrm{~kg}$

- Average human leg length: $81 \mathrm{~cm}=0.81 \mathrm{~m}$

The number varies according to country, race, age and gender; therefore, the value we use is just an approximation to present the general formula but does not apply to any specific group or individual.

Note: length $L$ is the combination of the length of human leg and the length of the model

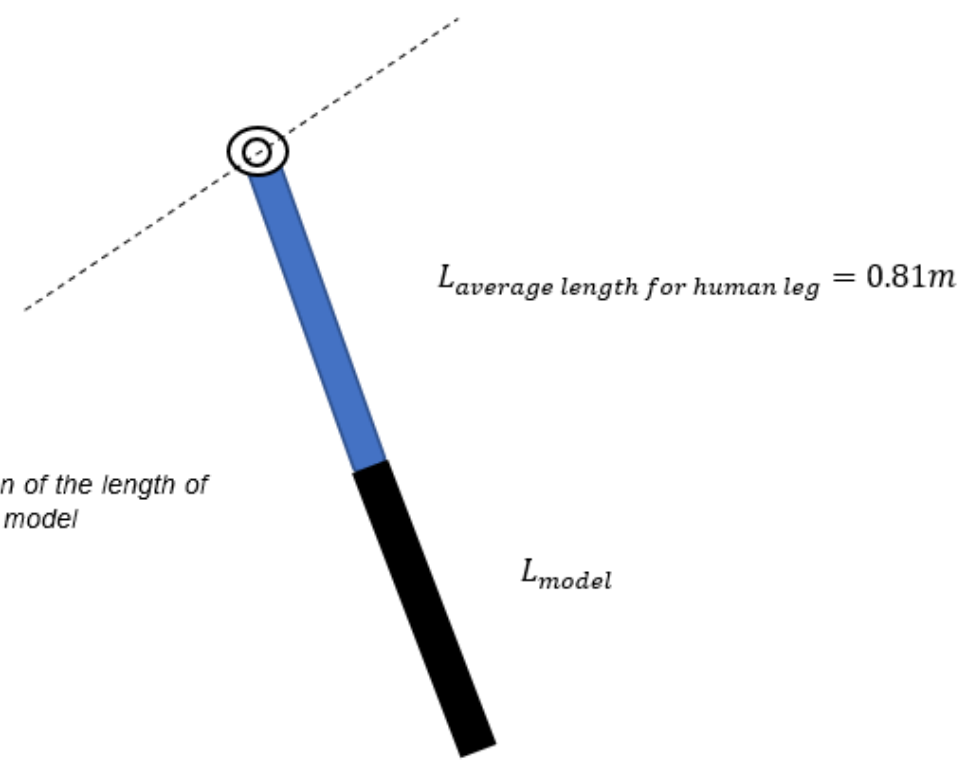

Figure 13: Combination between the model and human's leg

We have:

$$
\begin{aligned}
& L_{\text {total }}=L_{\text {leg }}+L_{\text {model }}=0.81 \mathrm{~m}+L_{\text {model }} \\
& m_{\text {total }}=m_{\text {leg }}+m_{\text {model }}=13.5 \mathrm{~kg}+m_{\text {model }}
\end{aligned}
$$

Correlation between length and mass of the model:

$$
\begin{aligned}
& m_{\text {model }}=\rho * V_{\text {model }} \\
& V_{\text {model }}=\pi r^{2} * L_{\text {model }}
\end{aligned}
$$

$\Rightarrow$ Mass of the model depends on its radius, length and material 
For the purpose of testing, we will use $\mathrm{r}=0.1 \mathrm{~m}, l=1 \mathrm{~m}$ and 3 different materials include wood, rubber and steel.

\begin{tabular}{|c|c|c|c|c|}
\hline Material & $\rho\left(\mathrm{kg} / \mathrm{m}^{3}\right)$ & $V_{\text {model }}\left(\mathrm{m}^{3}\right)$ & $L_{\text {total }}(\mathrm{m})$ & $m_{\text {model }}(\mathrm{kg})$ \\
\hline Wood & $600-700$ & 0.031 & 1.81 & 20.15 \\
\hline Rubber & 1522 & 0.031 & 1.81 & 47.18 \\
\hline Steel & 7750 & 0.031 & 1.81 & 240.25 \\
\hline
\end{tabular}

Table 1: Density, volume and mass for 3 different materials

Part 2: Analyze how the forces apply to the model for 2 cases (on the ground and under the water) and select the most effective material for the model.

Recall two fundamental torque equations:

$$
\begin{gathered}
\tau_{n e t}=\mathrm{I} \alpha \\
\tau_{n e t}=F r
\end{gathered}
$$

First of all, it is important to know how much torque the average person can exert through the hip joint, or in other words, how much force can make the quadriceps muscles handle without resulting in any injury. However, the torque at the hip joint depends on a variety of factors such as the speed that humans walk, other groups of muscles, the angle of knee flexion, etc. Moreover, we are not looking for the threshold of torque that can be produced by the hip joint but rather the average torque that a person exerts during the action of walking. Therefore, we will use an approximate number from the previous research that illustrates a reasonable torque when a human is walking. According to Reilley and Martens, the quadriceps muscle can take in more than $600 \mathrm{~kg}$ if the angle of knee flexion is over 130 degrees (graph provided in the Appendix). 
$\Rightarrow \tau \max =6000 \mathrm{~N}$

Set $\tau^{\prime}$ as the torque that human hip joint creates, range from 0 to $6000 \mathrm{~N} * \mathrm{~m}$

Set $\tau^{\prime \prime}$ as external torques created by the environment and applied on the leg in $\hat{\jmath}$ direction

$$
\Rightarrow \tau_{\text {net }}=\tau^{\prime}-\tau^{\prime \prime} \geq 0
$$

Set $\mu_{k 1}$ as the coefficient of kinetic friction for the surface of the ground.

We start by analyze forces for 2 cases, on the ground and under the water by calculating $\tau^{\prime \prime}$.

Ground: $\tau^{\prime \prime}=f_{k}=\mu_{k_{1}} * N$, where $\mu_{k_{1}}$ depends on the interaction between our model base material and the surface. Now we will use concrete for the surface on the ground and determine the material for the model base that minimizes the kinetic friction, which will lead to a reduction in $\tau^{\prime}$, the torque created by human hip joint.

Some examples for $\mu_{k_{1}}$ between concrete and different materials:

- Wood: 0.62

- Rubber: $0.60-0.85 \rightarrow 0.725$

- Horseshoe (steel): 0.58

\begin{tabular}{|c|c|c|c|}
\hline Material & $F_{N_{\text {total }}}(\mathrm{N})$ & $\mu_{k_{1}}$ & $f_{k}(\mathrm{~N})$ \\
\hline Wood & 565 & 0.62 & 350.3 \\
\hline Rubber & 830 & 0.725 & 601.75 \\
\hline Steel & 2722 & 0.58 & 1578.76 \\
\hline
\end{tabular}

Table 2: Gravitational force and kinetic friction for 3 different materials

Note: $F_{N_{\text {total }}}=$ half body mass + model mass

$\Rightarrow$ Conclusion: Wood or Rubber serve as better materials for the model since their weights and the frictional forces are less compared to Steel. Even though human hip joint is still 
able to handle frictional force created by Steel $(\tau \max =6000 \mathrm{~N})$ but it requires more energy to travel.

Water: $\tau^{\prime \prime}=\frac{1}{2} \rho v^{2} C_{D} A$, where $C_{D}$ is the drag coefficient based on the shape of the object immersed under water. For long cylinder, straight rod (which is the combination of the human leg with the model), the drag coefficient equals 0.82 . In the water, the frictional force will be small enough to be neglected and we therefore shift our attention to the drag force.

After a closer look, the drag force does not depend on its material but it is rather based on different factors as long as the volume of the object remains constant.

We have:

- Average velocity of the river: $0 \mathrm{~m} / \mathrm{s} \rightarrow 3.1 \mathrm{~m} / \mathrm{s} \sim 5.5 \mathrm{~km} / \mathrm{h}$

- Average human speed in the river: $2 \mathrm{~km} / \mathrm{h}$

- Velocity of the water relative to fluid: $5.5+2=7.5 \mathrm{~km} / \mathrm{h}$

- Since we set length of the model to be 1 meter (part 2-C), the length that under water is 0.8 meter

Cross-sectional Area of the model that immerses under water can be calculated by the length of the part immersed times diameter of the rod:

$\Leftrightarrow 0.8 * 0.1 * 2=0.16 \mathrm{~m}^{2}$

$\Leftrightarrow \tau^{\prime \prime}=\frac{1}{2} \rho v^{2} C_{D} A=\frac{1}{2} * 1000 * 7.5^{2} * 0.82 * 0.16=3690 \mathrm{~N}$

$\Rightarrow$ Conclusion: Material of the model does not affect the drag force applied to it. Moreover, the human hip joint can still overcome the drag force since the maximum torque it can create is $6000 \mathrm{~N}$, which is larger than $\tau^{\prime \prime}$.

Part 3: Finding the required torque $\left(\tau^{\prime}\right)$ that a human hip joint needs to create in order to achieve certain speed. This part mainly focuses on the moment of inertia and its equation that we calculated in part B - Calculating. 


$$
\tau=\mathrm{I} \alpha
$$

We utilize this equation in order to figure out how much torque should the human hip joint exert to achieve the ideal speed. Since the leg travels in rotational motion, we have to deal with the angular velocity $(\omega)$ and angular acceleration $(\alpha)$. However, the angle between the two legs is small enough due to the length of the model and the leg combined (1.81 meter), we can consider $v$ as $\omega$ and $a$ as $\alpha$.

For a person, to start at rest $\left(v_{0}=0\right)$ and achieve $5 \mathrm{~km} / \mathrm{h}\left(v_{f}=5\right)$ in 0.5 second, the average acceleration is:

$a_{\text {average }}=\frac{v_{f}-v_{0}}{t}=\frac{5}{0.5}=10 \mathrm{~m} / \mathrm{s}^{2}$

Calculating $\tau^{\prime}$ with different values for $b, h$ and $m$ (with height $=1 / 2$ base)

- Rectangular prism

$\frac{m b^{2}}{3}+\frac{m h^{2}}{12}+\frac{m h^{4}}{4}+\frac{4 m L^{4}}{3}+m h^{2} L$

\begin{tabular}{|c|c|c|c|c|}
\hline $\mathrm{L}(\mathrm{m})$ & $\mathrm{m}(\mathrm{kg})$ & $\mathrm{b}(\mathrm{m})$ & $\mathrm{h}(\mathrm{m})$ & $\tau^{\prime}(N)=\mathrm{I} \alpha$ \\
\hline 1.81 & 5 & 0.1 & 0.05 & 715.93 \\
\hline 1.81 & 5 & 0.2 & 0.1 & 717.14 \\
\hline 1.81 & 5 & 0.3 & 0.15 & 719.16 \\
\hline 1.81 & 10 & 0.4 & 0.2 & 1443.99 \\
\hline 1.81 & 10 & 0.5 & 0.25 & 1451.31 \\
\hline 1.81 & 10 & 0.6 & 0.3 & 1460.29 \\
\hline
\end{tabular}

Table 3: Mass, base and height of the rectangle prism to calculate the applied torque 
- Triangular prism

$\frac{4 b^{3} m}{3 h}+\frac{2 b h m}{3}-\frac{b^{2}+h^{2}}{36}+\frac{4 m L h}{3}+\frac{4 m L^{2}}{3}-\frac{25 b^{2} m}{18}+\frac{5 m h^{2}}{18}$

\begin{tabular}{|c|c|c|c|c|}
\hline $\mathrm{L}(\mathrm{m})$ & $\mathrm{m}(\mathrm{kg})$ & $\mathrm{b}(\mathrm{m})$ & $\mathrm{h}(\mathrm{m})$ & $\tau^{\prime}(N)=\mathrm{I} \alpha$ \\
\hline 1.81 & 5 & 0.1 & 0.05 & 225.28 \\
\hline 1.81 & 5 & 0.2 & 0.1 & 233.82 \\
\hline 1.81 & 5 & 0.3 & 0.15 & 244.04 \\
\hline 1.81 & 10 & 0.4 & 0.2 & 511.91 \\
\hline 1.81 & 10 & 0.5 & 0.25 & 539.07 \\
\hline 1.81 & 10 & 0.6 & 0.3 & 569.59 \\
\hline
\end{tabular}

Table 4: Mass, base and height of the rectangle prism to calculate the applied torque

$\Rightarrow$ Conclusion: When achieving ideal speed and acceleration, human hip joint can exert more than enough force that it is required since the maximum torque it can possibly create without cause any injuring is approximately $6000 \mathrm{~N}$. 


\section{DISCUSSION}

The purpose of this experiment is to analyze the imitated prosthetic leg model. Through different values of the input data, it shows the maximum torque that a human leg creates in order to overcome forces in nature such as the static friction and drag forces, and even with the weight of the model hanging on the leg. The quadriceps muscles can perform with only a small portion of the force and energy to achieve the ideal velocity $(5 \mathrm{~km} / \mathrm{h})$, which is the average walking speed of a human. The drag force is the only force that stands out from the others, which requires approximately $3690 \mathrm{~N}$ to achieve the ideal speed. Generally, the result is expected. In real life, when we walk on the ground or under water, we feel resistant forces that have opposite directions with the path we are moving; however, most of the time, we are able to overcome those forces without any difficulty. Making the problem more complicated, we can have a more detailed look at the forces coming into play, since attaching the model which includes not only the rod but also the base requires us to calculate the forces in different places. Moreover, we have to take torque into account by using a variety of methods such as the parallel axis theorem or finding the moment of inertia for the 3D object. We are then able to determine the two general equations for the rectangle and triangular prisms based on their masses, lengths, and heights. Then, we test different values in order to compare them with each other. Surprisingly, the change in the base and height does not affect much the torque that hip joint requires in order to exert the force. However, the change in mass (which could be caused by the change in material and followed by the increase in density) increases the required torque significantly. The result of this paper solidifies the ideas about forces that have been studied for many years in the engineering field, and also provides a different approach to analyzing of the force problems. 


\section{CONCLUSION AND RECOMMENDATIONS}

The paper provides 3 major parts about illustrating the impact of forces on a moving object and comparing the nature forces with torque that is created by the human hip joint. By not only considering translational but also rotational motion, it analyzes a variety of forces and torques and many other aspects that go along with it. Through introducing and examining the simple prosthetic leg model, we have an idea of how forces work in nature. The purpose of parts A and $\mathrm{B}$ is to provide the general equations regarding the many unknown values of the problem, such as the mass of the human, dimensions of the model, the ideal velocity, etc. However, those values are controllable, which means they do not depend on the environment but rather can be adjusted by humans. On the other hand, in part $\mathrm{C}$, we take a closer look into numerical values and examine the functionality of the model. Eventually, the data proves that the torque created by the human hip joint can overcome most of the forces in nature. The experiment can be analyzed even further and give a more insightful outcome to future practitioners who are interested in this project. They may take several other factors, hereby neglected, into account such as calculating the torque in the human knee joint. 
Undergraduate Journal of M athematical M odeling: One +Two, Vol. 10, Iss. 2 [2020], Art. 2

\section{NOMENCLATURE}

\begin{tabular}{|c|c|c|}
\hline Symbol & Meaning & Units \\
\hline$F_{B}$ & Buoyancy force & $\mathrm{N}$ \\
\hline$F_{D}$ & Drag force & $\mathrm{N}$ \\
\hline$C_{D}$ & Drag coefficient & N/A \\
\hline$\rho$ & Density & $\mathrm{kg} / \mathrm{m}^{3}$ \\
\hline$\tau$ & Torque & $\mathrm{N} * \mathrm{~m}$ \\
\hline$\tau \max$ & Maximum torque that human hip & $\mathrm{N} * \mathrm{~m}$ \\
\hline & joint can create & \\
\hline$\tau^{\prime}$ & Torque that hip joint creates & $\mathrm{N} * \mathrm{~m}$ \\
\hline$\tau^{\prime \prime}$ & External torque & $\mathrm{N}^{*} \mathrm{~m}$ \\
\hline$\omega$ & Angular velocity & $\theta / s$ \\
\hline$\alpha$ & Angular acceleration & $\theta / s^{2}$ \\
\hline$f_{k}$ & Kinetic friction & $\mathrm{N}$ \\
\hline$\mu_{k_{1}}$ & Coefficient of kinetic friction on & N/A \\
\hline & concrete & \\
\hline$L$ & Length of the combination between & $\mathrm{m}$ \\
\hline & the model and the human leg & \\
\hline CoM & Center of mass & N/A \\
\hline
\end{tabular}




\section{REFERENCES}

Body Segment Data. (n.d.). Retrieved December 12, 2019, from

https://exrx.net/Kinesiology/Segments.

Chakravarthy, K., Sultana, M. S., Srinath, A., \& Kumar, N. (2017). A mathematical model to determine the torque for a prosthetic leg - Lagrangian equation. Retrieved December 12, 2019, from https://acadpubl.eu/jsi/2017-116-5-7/articles/7/9.pdf.

DASSY professional workwear: HOW TO ... choose the right inside leg length. (n.d.). Retrieved from https://www.dassy.eu/pt/About_us/News/the-right-inside-leg-length/.

Donald T. Reilly \& Marc Martens (1972) Experimental Analysis of the Quadriceps Muscle Force and Patello-Femoral Joint Reaction Force for Various Activities, Acta Orthopaedica Scandinavica, 43:2, 126-137, DOI: $\underline{10.3109 / 17453677208991251}$

Drag coefficient. (2019, November 15). Retrieved December 12, 2019, from https://en.wikipedia.org/wiki/Drag_coefficient.

Elert, G. (n.d.). Speed of a River. Retrieved December 12, 2019, from https://hypertextbook.com/facts/2006/NervanaGaballa.shtml

Normal weight ranges: Body mass index (BMI). (n.d.). Retrieved December 12, 2019, from https://www.cancer.org/cancer/cancer-causes/diet-physical-activity/body-weight-andcancer-risk/adult-bmi.html 


\section{APPENDIX}

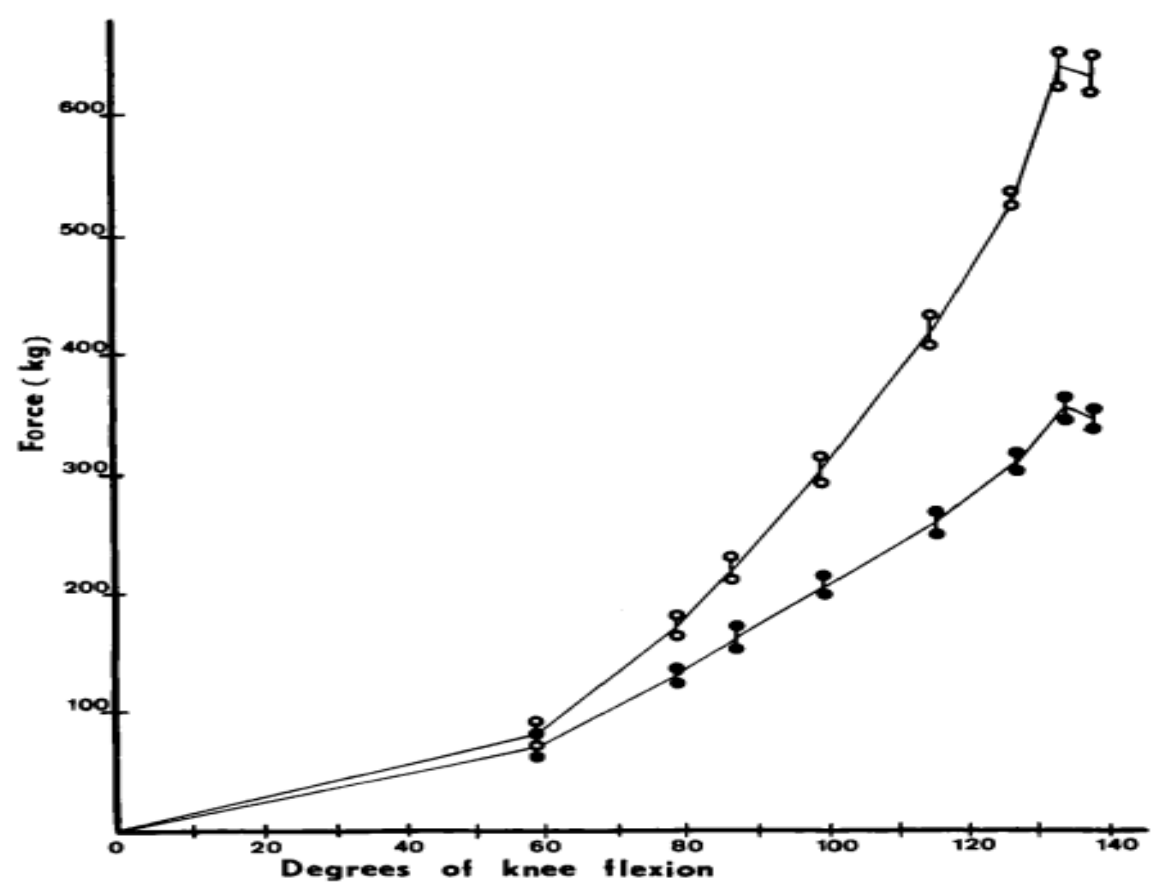

Figure 1: Amount of force is created by human hip joint based on degrees of knee flexion

TABLE 2 Adult BMI Chart

\begin{tabular}{|c|c|c|c|c|c|c|c|c|c|c|c|c|c|c|c|c|c|}
\hline BMI & 19 & 20 & 21 & 22 & 23 & 24 & 25 & 26 & 27 & 28 & 29 & 30 & 31 & 32 & 33 & 34 & 35 \\
\hline \multicolumn{8}{|c|}{ Height } & \multicolumn{10}{|c|}{ Weight in Pounds } \\
\hline $4^{\prime} 10^{\circ}$ & 91 & 96 & 100 & 105 & 110 & 115 & 119 & 124 & 129 & 134 & 138 & 143 & 148 & 153 & 158 & 162 & 167 \\
\hline $4^{\prime} 11^{*}$ & 94 & 9 & 104 & 109 & 114 & 119 & 124 & 128 & 133 & 138 & 143 & 148 & 153 & 156 & 163 & 168 & 173 \\
\hline $5^{\prime}$ & 97 & 102 & 107 & 112 & 118 & 123 & 128 & 133 & 138 & 143 & 148 & 153 & 158 & 163 & 160 & 174 & 179 \\
\hline $5^{\prime \prime} 1{ }^{\prime \prime}$ & 100 & 106 & 111 & 116 & 122 & 127 & 132 & 137 & 143 & 148 & 153 & 158 & 164 & 169 & 174 & 100 & 185 \\
\hline $52^{*}$ & 104 & 109 & 115 & 120 & 126 & 131 & 136 & 142 & 147 & 153 & 158 & 164 & 169 & 175 & 180 & 106 & 191 \\
\hline $53^{\circ}$ & 107 & 113 & 118 & 124 & 130 & 135 & 141 & 146 & 152 & 158 & 163 & 169 & 175 & 180 & 160 & 191 & 197 \\
\hline $5^{\prime} 4^{\prime \prime}$ & 110 & 116 & 122 & 128 & 134 & 140 & 145 & 151 & 157 & 163 & 169 & 174 & 180 & 186 & 198 & 197 & 204 \\
\hline $55^{\circ}$ & 114 & 120 & 126 & 132 & 138 & 144 & 150 & 156 & 162 & 168 & 174 & 180 & 106 & 192 & 198 & 204 & 210 \\
\hline $56^{\circ}$ & 118 & 124 & 130 & 136 & 142 & 148 & 155 & 161 & 167 & 173 & 179 & 186 & 192 & 196 & 204 & 210 & 216 \\
\hline $57^{\circ}$ & 121 & 127 & 134 & 140 & 146 & 153 & 159 & 166 & 172 & 178 & 185 & 191 & 196 & 204 & 211 & 217 & 223 \\
\hline $58^{\circ}$ & 125 & 131 & 136 & 144 & 151 & 158 & 164 & 171 & 177 & 184 & 190 & 197 & 200 & 210 & 216 & 223 & 230 \\
\hline $59^{\circ}$ & 128 & 136 & 142 & 149 & 155 & 162 & 169 & 176 & 182 & 189 & 196 & 200 & 209 & 216 & 223 & 290 & 236 \\
\hline $5^{\prime} 10^{\circ}$ & 132 & 139 & 146 & 153 & 160 & 167 & 174 & 181 & 188 & 195 & 202 & 209 & 216 & 222 & 229 & 246 & 243 \\
\hline $5^{\prime} 11^{\circ}$ & 136 & 143 & 150 & 157 & 165 & 172 & 179 & 186 & 193 & 200 & 208 & 215 & 222 & 229 & 236 & 243 & 250 \\
\hline $6^{\prime}$ & 140 & 147 & 154 & 162 & 169 & 177 & 184 & 191 & 199 & 206 & 213 & 221 & 228 & 235 & 242 & 250 & 258 \\
\hline $6^{\prime \prime} 1^{\prime \prime}$ & 144 & 151 & 159 & 166 & 174 & 182 & 189 & 197 & 204 & 212 & 219 & 221 & 235 & 242 & 250 & 257 & 265 \\
\hline $62^{\circ}$ & 148 & 155 & 163 & 171 & 179 & 186 & 194 & 202 & 210 & 218 & 225 & 233 & 241 & 249 & 256 & 264 & 272 \\
\hline \multirow[t]{2}{*}{$63^{\circ}$} & 152 & 160 & 168 & 176 & 184 & 192 & 200 & 208 & 216 & 224 & 232 & 240 & 248 & 256 & 254 & 272 & 279 \\
\hline & \multicolumn{6}{|c|}{ Healthy Weight } & \multicolumn{5}{|c|}{ Overweight } & & & & & & \\
\hline
\end{tabular}

Table 1: Normal weight ranges: Body mass index (BMI) 
Percentages of Total Body Weight

\begin{tabular}{|l|l|l|l|}
\hline \multicolumn{1}{|c|}{ Segment } & Males & Females & Averag \\
\hline Head & 8.26 & 8.2 & 8.23 \\
\hline Whole Trunk & 55.1 & 53.2 & 54.15 \\
\hline Thorax & 20.1 & 17.02 & 18.56 \\
\hline Abdomen & 13.06 & 12.24 & 12.65 \\
\hline Pelvis & 13.66 & 15.96 & 14.81 \\
\hline Total Arm & 5.7 & 4.97 & 5.335 \\
\hline Upper Arm & 3.25 & 2.9 & 3.075 \\
\hline Forearm & 1.87 & 1.57 & 1.72 \\
\hline Hand & 0.65 & 0.5 & 0.575 \\
\hline Forearm \& Hand & 2.52 & 2.07 & 2.295 \\
\hline Total Leg & 16.68 & 18.43 & 17.555 \\
\hline Thigh & 10.5 & 11.75 & 11.125 \\
\hline Leg & 4.75 & 5.35 & 5.05 \\
\hline Foot & 1.43 & 1.33 & 1.38 \\
\hline Leg \& Foot & 6.18 & 6.68 & 6.43 \\
\hline
\end{tabular}

Table 2: Percentage of body parts compared to the whole body 


\begin{tabular}{|lcl|}
\hline & Kinetic & Static \\
Rubber on concrete (dry) & 0.68 & 0.90 \\
Rubber on concrete (wet) & 0.58 & \\
Rubber on asphalt (dry) & 0.67 & 0.85 \\
Rubber on asphalt (wet) & 0.53 & \\
Rubber on ice & 0.15 & \\
Waxed ski on snow & 0.05 & 0.14 \\
Wood on wood & 0.30 & 0.42 \\
Steel on steel & 0.57 & 0.74 \\
Copper on steel & 0.36 & 0.53 \\
Teflon on Teflon & 0.04 & \\
\hline
\end{tabular}

Table 3: Short version for coefficient of kinetic friction between different materials

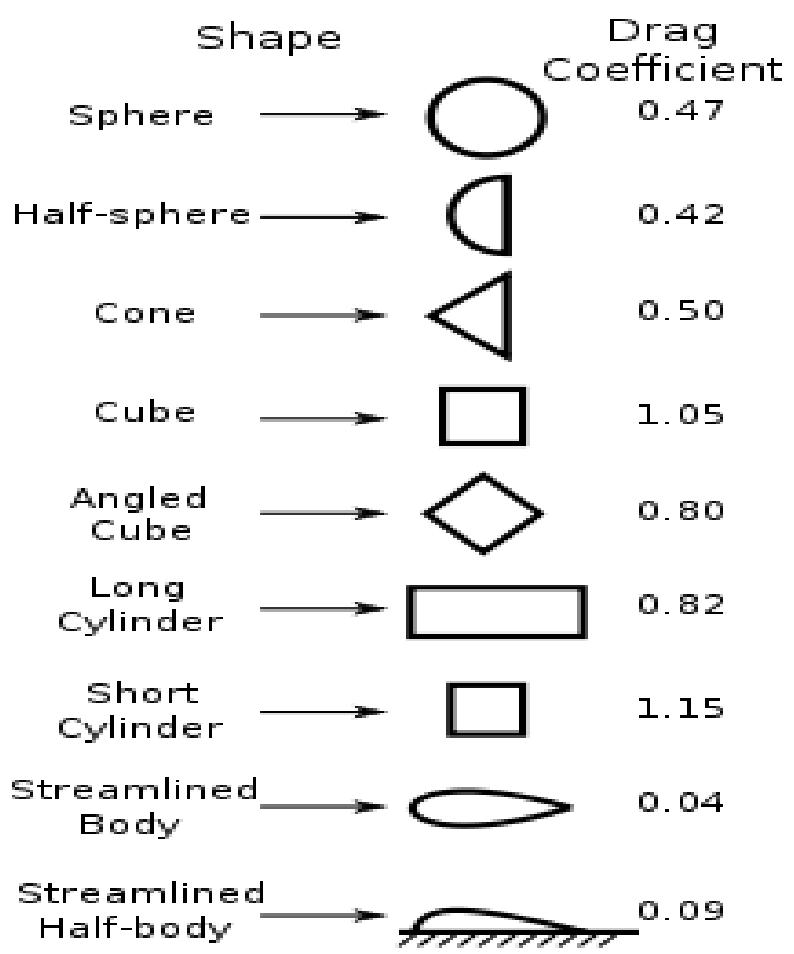

Measured Drag Coefficients

Table 4: Short version for drag coefficient based on different shapes 